\title{
Soft X-ray emission as diagnostics for Maunder minimum stars
}

\author{
Katja Poppenhaeger and Jürgen H. M. M. Schmitt \\ Hamburger Sternwarte, Gojenbergsweg 112, 21029 Hamburg, Germany \\ email: katja.poppenhaeger@hs.uni-hamburg.de
}

\begin{abstract}
The identification of stars in a Maunder minimum state purely from their chromospheric emission (for example in Ca II lines) has proven to be difficult. Photospheric contributions, metallicities and possible deviations from the main sequence stage may lead to very low values of the traditional chromospheric activity indicators, while no Maunder minimum state may be present. X-ray observations can be a key tool for identifying possible Maunder minimum stars: We have detected very soft X-ray emission from low-temperature coronal plasma, similar to emission from solar coronal holes, in several stars with very low chromospheric activity indicators. The coronal properties inferred from X-ray observations can therefore yield a crucial piece of information to verify Maunder minimum states in stars.
\end{abstract}

Keywords. X-rays: stars, stars: activity, stars: coronae, stars: late-type

\section{Maunder minimum candidates}

We know of three phases in the life of the Sun when it displayed very low activity over a long time: the Maunder, Dalton and Spörer Minima, of which the Maunder Minimum was the most extreme. The causes of such Grand minima, however, are still unknown. The recent very low and slightly prolonged minimum of the solar cycle in 2008/2009 may have given us a taste of what a Maunder-minimum state might look like observationally. By observing other stars, we can estimate how frequent such extended minima states are. Initially, a large number of stars $(30 \%$, later corrected to $10-15 \%)$ was assumed to be in a Maunder minumum based on chromospheric activity measured in the Mount Wilson project (Saar \& Baliunas 1992). However, it was shown by Wright (2004) that most of these stars were actually evolved stars, making their chromospheric activity indices incomparable to main sequence stars. Additionally, chromospheric activity is always influenced by a small amount of basal heating, making X-ray and EUV measurements necessary to truly assess if a star is in a Maunder minimum (Judge \& Saar 2007).

We have started an observational program at X-ray wavelengths to determine the coronal properties of several solar-like stars with very low chromospheric activity, in order to identify possible Maunder minimum stars. We demonstrate here our technique to determine coronal temperatures for inactive stars, and present the derived temperatures for $51 \mathrm{Peg}$ and, as a moderate activity comparison, HD 189733.

\section{Inferring coronal temperatures}

Stellar (and solar) X-ray emission is a direct consequence of the magnetic heating of coronae, and is therefore an ideal tool to measure magnetic activity. It is well known that both X-ray luminosities and coronal temperatures are correlated with the activity level; for quiet regions on the Sun, coronal temperatures of approximately $1 \mathrm{MK}$ have 

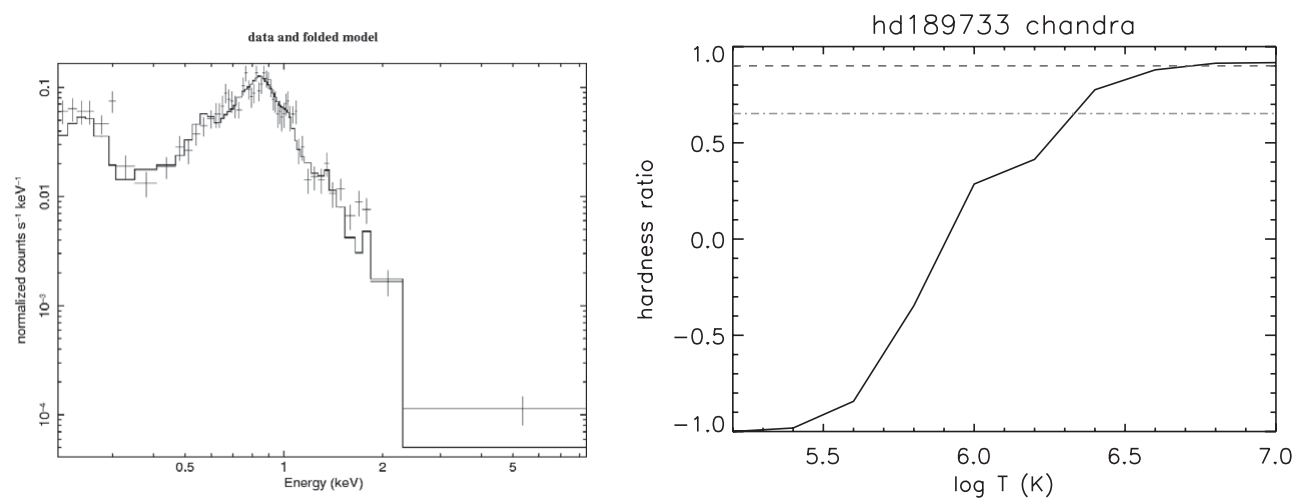

Figure 1. Left panel: Soft X-ray spectrum of the moderately active star HD 189733A. The spectral fit yields a mean coronal temperature of $\log T=6.59$. Right panel: Theoretical temperature-dependent hardness ratio (black solid) together with the observed hardness ratio of HD 189733A, measured from only 20 source counts (green dashed) with $1 \sigma$ errors (green dashdotted).

been measured, while more active stars can easily display coronal temperature components above $10 \mathrm{MK}$. Consequently, stars with a very low activity level such as Maunder minimum candidates will display low X-ray luminosities and coronal temperatures.

Furthermore, an estimate of the coronal temperature is needed to convert a measured $\mathrm{X}$-ray count rate into the stellar X-ray flux. For X-ray bright stars, moderately resolved CCD spectra or even high-resolution grating spectra with sufficient signal to noise ratio can be collected within reasonable exposure times. These spectra can be fitted with an optically-thin thermal plasma model to yield coronal temperatures and emission measures. For low-activity stars, however, one often collects only 20 or fewer source counts, and therefore needs other methods to infer the coronal temperature.

A convenient way to derive an estimate for the coronal temperature of an X-ray dim target is to analyze hardness ratios. A hardness ratio is defined as $H R=\frac{H-S}{H+S}$, with $H$ and $S$ being the number of X-ray photons in a predefined hard and soft energy band. As stellar X-ray spectra shift to lower energies for lower coronal temperatures, hardness ratios are intrinsically temperature-dependent. To demonstrate the accuracy of this method, we compare the coronal temperature estimate for the moderately active $\mathrm{K}$ star HD 189733A derived from its X-ray CCD spectrum, consisting of ca. 1200 source counts, as opposed to the temperature derived from the hardness ratio of a short excerpt of the data with only 20 source counts in total.

HD 189733A was observed by the Chandra X-ray telescope with the ACIS-S camera for $20 \mathrm{ks}$ on July 05 2011. The stellar emission is soft, with negligible flux above $5 \mathrm{keV}$. The X-ray spectrum in the 0.2-5 keV energy band is shown in Fig. 1 (left panel), together with a two-temperature-component fit (details of the fit given in Table 1).

We then analyze a fraction from that data which contains only 20 source counts. We calculate the theoretical hardness ratios of synthetic spectra folded with the ACIS-S response using Xspec v.12 and compare them to the measured hardness ratio, using energy ranges of $200-350 \mathrm{eV}$ and $350-1000 \mathrm{eV}$ as the soft and hard band, respectively. The threshold between the hard and soft band is at a quite low energy; this is because we will need the low-energy diagnostic power when investigating the soft X-ray emission of low-activity stars. For consistency, we use the same energy threshold for HD 189733A as well, even if a higher threshold would reduce the error bars for this moderately active star. The result of our analysis is shown in Fig. 1 (right panel). The observed hardness 
Table 1. Coronal properties of HD 189733A, derived from ACIS-S CCD spectra; emission measures given in units of $10^{50} \mathrm{~cm}^{-3}$.

\begin{tabular}{ccccccc}
\hline$T_{1}(\mathrm{~K})$ & $E M_{1}$ & $\log T_{2}(\mathrm{~K})$ & $E M_{2}$ & mean $\log T(\mathrm{~K})$ & $\chi^{2}$ & $L_{X}(\mathrm{erg} / \mathrm{s})$ \\
\hline 6.14 & 4.82 & 6.84 & 4.03 & 6.59 & 1.44 & $1.25 \times 10^{28}$ \\
\hline
\end{tabular}
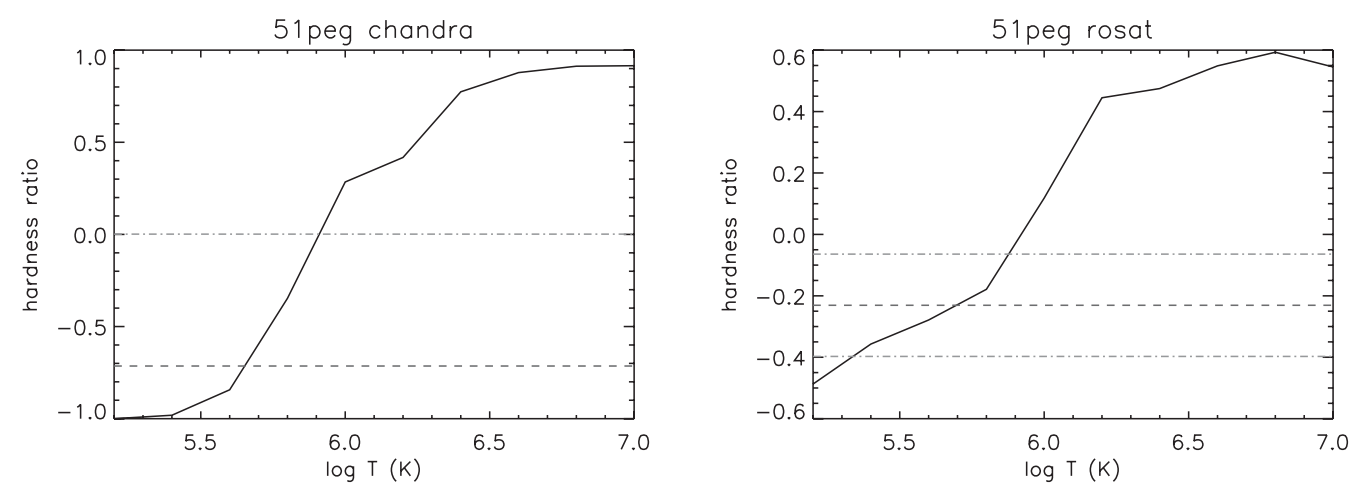

Figure 2. Left panel: As Fig. 1 (right panel), but for the Maunder minimum candidate $51 \mathrm{Peg}$. The star clearly displays a mean coronal temperature below $1 \mathrm{MK}$ during the Chandra observation in 2008. Right panel: Same as left panel, but for archival X-ray data from ROSAT, taken in 1992. The very low coronal temperature seems to be persistent over 16 years.

ratio matches the synthetic spectra at a coronal temperature of $\log T=6.65$, which is in close agreement with the spectral fit to the full data; however, due to the low energy threshold, it not well constrained towards higher temperatures.

With the validity of the method shown, we can apply it to low activity stars to determine their coronal temperature. The G-type star 51 Peg, host star to a Hot Jupiter, displays a very low magnetic activity level in chromospheric measurements. Its mean Mount-Wilson S index is 0.149 (Baliunas et al. 1995); for comparison, the Sun's S index varies between ca. 0.16 and 0.21 during its activity cycle. In a 5 ks observation with Chandra ACIS-S performed in 2008, we collected eight X-ray source photons from 51 Peg. Additionally, an archival ROSAT observations from 1992 exists with an exposure time of 12.5 ks. In Fig. 2 we show the observed and theoretical hardness ratios; we can constrain 51 Peg's mean coronal temperature to be $\log T \leqslant 5.85$ during the Chandra exposure and to $5.35 \leqslant \log T \leqslant 5.9$ during the ROSAT pointing. The X-ray activity indicator $\log L_{X} / L_{b o l}$ has a low value of $\approx-7$ (Poppenhäger et al. 2009).

Putting these coronal temperatures into the solar context is somewhat complicated by the fact that disk-integrated soft X-ray spectra of the Sun are difficult to construct. However, quiet regions on the Sun seem to be quite well characterized by a coronal temperature of $\approx 1 \mathrm{MK}$ (Orlando et al. 2001). This puts 51 Peg's corona at the lowest end of the solar coronal temperature span. The very low coronal temperature and $L_{X} / L_{b o l}$ ratio which are persistent over a long time span suggest that $51 \mathrm{Peg}$ may indeed be in a Maunder minimum state.

The case of $51 \mathrm{Peg}$ indicates that coronae during Maunder minima might observationally not be very different from the solar corona in deep single minima like in 2008/2009, except for the longer duration. Already the compilation by Schmitt (1997) of X-ray fluxes normalized by the stellar surface showed that even in very low-activity stars, the X-ray surface flux does not fall below $10^{4} \mathrm{erg} \mathrm{s}^{-1} \mathrm{~cm}^{-2}$ in the 0.1-2.4 energy band. Apparently, coronae experience a low level of heating even during extended minima. 


\section{Conclusion}

With contemporaneous X-ray telescopes, coronal temperature estimates can be inferred even for very inactive cool stars. Our observations show that the planet-hosting star 51 Peg is probably in a Maunder minimum state, characterized by low coronal temperature as well as low X-ray and chromospheric activity indicators over a time span of 16 years. Even in such a long-term minimal activity state, the coronal X-ray emission does not vanish completely; it is similar to the solar coronal emission of quiet regions, indicating that long-term minima may not be fundamentally different from single deep minima of the solar cycle. X-ray observations of a larger sample of inactive stars are under way and will yield more insight into the frequency of extended minimium states in solar-like stars.

\section{References}

Baliunas, S. L., Donahue, R. A., Soon, W. H., et al. 1995, ApJ, 438, 269

Judge, P. G. \& Saar, S. H. 2007, ApJ, 663, 643

Orlando, S., Peres, G., \& Reale, F. 2001, ApJ, 560, 499

Poppenhäger, K., Robrade, J., Schmitt, J. H. M. M., \& Hall, J. C. 2009, A\&\&A, 508, 1417

Saar, S. H. \& Baliunas, S. L. 1992, in Astron. Soc. Pacific CS, Vol. 27, The Solar Cycle, ed. K. L. Harvey, 150-167

Schmitt, J. H. M. M. 1997, A\& $A, 318,215$

Wright, J. T. 2004, AJ, 128, 1273

\section{Discussion}

Dibyendu NAndy: From the point of view of dynamo theory it is important to know whether stars fall in Maunder minima suddenly or gradually. Is there any observation like this from other stars that can tell us about it?

Katja Poppenhaeger: It's very difficult to obtain information on transitions from cyclic activity to a Grand Minimum or the other way around from X-ray observations. Usually, our time sampling is rather sparse and we are somewhat limited by the life time of the X-ray satellites (even if cross-calibration with other missions like ROSAT works rather well), so that X-ray data cannot yield strong constraints on such transitions at the moment.

MARK Giampapa: Low activity stars are not just low in X-ray luminosity but also low in coronal temperature. Have you thought about a cause for the structure of the corona of these stars?

Katja Poppenhaeger: Also our sample stars follow the canonical picture of low coronal temperature being correlated with low overall X-ray luminosity, My feeling is that the coronae of these stars might consist in a large part of coronal holes, and very small loops in the remaining parts. However, with our few X-ray source counts, we cannot perform the sophisticated analysis needed to really infer the coronal structure.

VOLKER BothmeR: What definition of coronal temperature do you use?

Katja Poppenhaeger: In my analysis, I use the average coronal temperature inferred from the X-ray emission between 0.15 and $2 \mathrm{keV}$. This is a very strong simplification; when analyzing high signal-to-noise stellar X-ray spectra, one usually needs several temperature components for an adequate fit of the coronal spectra. For the low X-ray signal of our sample stars, however, the mean coronal temperature is the best one can do. 